\title{
Erratum
}

\section{Charges in Spacelike Cones}

Claudio d'Antoni and Klaus Fredenhagen

Centre de Physique Théorique, C.N.R.S. - Luminy - Case 907, F-13288 Marseille Cedex 9, France

Commun. Math. Phys. 94, 537-544 (1984)

The proof of Proposition 2.2 relies on an incorrect statement on the test function $H_{R^{\prime} K^{\mu_{4}}}^{u_{1}}$. A correct proof may be obtained by the following argument. Let $R^{\prime}=2 R$. Then the statement on the vanishing of the right-hand side of inequality (*) in the limit $R \rightarrow \infty$ is true. Moreover, the limit is approached faster than by any power of $R$. For the second term this follows by the argument given in the proof, for the first term one applies the methods which have been used in the proof of Proposition 2.1. The proposition then follows by summing the series $\sum_{k=0}^{\infty}\left\|\left(Q_{S, 2^{k+1} R}-Q_{S, 2^{k} R}\right) A \Omega\right\|$.

Communicated by R. Haag

Received October 23, 1984 